\title{
Extended Field Postoperative Radiotherapy (EFRT) Yields better Outcomes as Compared to Localised Field RT (LFRT) in Radically Resected Gallbladder Cancer (GBC)
}

\author{
Sushma Agrawal ${ }^{1}$, Rajan Saxena ${ }^{2}$ \\ ${ }^{1}$ Department of Radiotherapy, Sanjay Gandhi Post Graduate Institute of Medical Sciences, Lucknow, India. ${ }^{2}$ Department of Surgical \\ Gastroenterology, Sanjay Gandhi Post Graduate Institute of Medical Sciences, Lucknow, India.
}

\begin{abstract}
Background: Postoperative adjuvant RT is recommended in node positive and positive resection margins patients after radical surgery (RS) in GBC. However there are no guidelines to suggest the regions to be irradiated. Our aim is to compare whether EFRT or LFRT improves outcomes. Methods and Materials: Records of GBC patients who underwent adjuvant chemo-radiotherapy after RS, between January 2007 and December 2014 were reviewed. Demographic data, histopathological data, target delineation, RT treatment planning details, concurrent chemotherapy, side-effects of treatment, and survival data were collated. Patients were treated with either of two techniques: Localised field RT [LFRT] [from 2007-2010] or Extended field RT [EFRT] [from 2011-2014] along with concurrent 5-FU/capecitabine. LFRT involved RT to GB bed, peri-portal, common hepatic artery (CHA) and coeliac lymph nodes and EFRT involved RT to GB bed, peri-portal, CHA, coeliac, gastro-duodenal, superior mesenteric and para aortic lymph nodes. The RT dose was $50.4 \mathrm{~Gy} / 28$ fractions $/ 5.5$ weeks. Loco-regional recurrence rate (LRR), Overall (OS) and Disease free Survival (DFS) was computed with Kaplan Meier method. Results: Out of 60 patients reviewed, 30 were treated with EFRT and 30 with LFRT. There was no significant difference in the acute and late side-effects between the two techniques. At a median follow-up of 44 months (range 36-120 months), $37.5 \%$ patients developed LRR ( $13.3 \%$ vs $40 \%$ in EFRT and LFRT, $\mathrm{p}=\mathrm{NS}$ ). The median OS was not reached (NR) vs 42 months and the median DFS was NR vs 30 months in EFRT vs LFRT respectively ( $p=0.01$ and 0.016 ). The 5 year OS was $80 \%$ vs $42 \%$ and 5 year DFS was $80 \%$ vs $40 \%$ for EFRT and LFRT respectively ( $\mathrm{p}=0.01$ and 0.016 ). Conclusions: Based on our findings, we conclude that EFRT reduces LRR and improves survival in patients with absence of lymphovascular space invasion (LVI) and perineural invasion (PNI). This observation is hypothesis generating and merits validation in a randomised study.
\end{abstract}

Keywords: Adjuvant- postoperative radiotherapy- target volume- gallbladder cancer

Asian Pac J Cancer Care, 5 (4), 287-293

\section{Introduction}

GBC has a poor prognosis due to its highly aggressive behaviour, with only $10 \%$ cases presenting as resectable disease [1]. Radical surgery (RS) is the standard of care. Loco-regional recurrence (LRR) after RS has been reported in the hepatic hilum, bilio-enteric anastomosis, liver resection margins and retro-portal lymph nodes [2]. To prevent recurrences, adjuvant chemo-radiotherapy is advocated in node positive, resection margin positivity (R1) and high risk features like lympho-vascular
Submission Date: 08/21/2020Ａcceptance Date: 11/07/2020

\footnotetext{
Corresponding Author:

Dr. Sushma Agrawal

Department of Radiotherapy, Sanjay Gandhi Post Graduate Institute of Medical Sciences, Lucknow, India.

Email: sushmaagrawal@yahoo.co.uk
} 
from inclusion of gallbladder bed and regional lymph nodes (peri-portal, celiac, pancreatico-duodenal with or without para aortic nodes) to whole abdomen irradiation with a boost to the tumour bed [6-8]. LRR with irradiation of para aortic nodes is $16 \%$ versus $27 \%$ when para aortic nodes were not irradiated and $34 \%$ with whole abdominal irradiation [6-7-9]. Recently a systematic review of surgical-pathological series on GBC, reported that the incidence of various lymph-node station involvement in GBC is $25 \%$ (peri-choledochal), $23 \%$ along cystic duct, $17 \%$ (retro-portal lymph nodes), $15.8 \%$ along common hepatic artery, $13.4 \%$, along the hepatoduodenal ligament, $12.3 \%$ retro-pancreatic nodes, $7.7 \%$ anterior and posterior pancreaticoduodenal nodes, $6 \%$ para-aortic and $5.1 \%$ for hilar lymph nodes. They suggested that these lymph-node groups should be irradiated in GBC [10].

With a view to get clarity on the extent of lymph node (LN) irradiation required, we reviewed our departmental data of postoperative GBC patients who received chemoradiation (CTRT) during the period 2007-2014. Localised field RT (LFRT, Figure1)) [practised from 2007-2010] was one where the target volume included GB bed and LN (along peri-portal, common-hepatic artery, coeliac artery: abdominal LN stations 5, 6, 9 and 12). Extended field (EFRT, Figure 1) [practised from 2011-2014] was one where the target volume also included the superior mesenteric artery and pre and para-aortic nodes (abdominal LN station 5, 6, 8, 9, 12, 13, 16) in addition to LFRT. In the absence of any delineation guidelines for GBC, EFRT was initiated in the department in 2011, after the availability of RTOG Atlas for target volume delineation in pancreatic cancers [11].

\section{Materials and Methods}

After SGPGI institutional ethics committee approval, the records of GBC patients who received CTRT after RS (January 2007 and December 2014) were retrospectively reviewed. The need for informed consent was waived off by the ethics committee. All methods were performed in accordance with the relevant guidelines and regulations. Demographic and histopathological data, CTV, treatment planning data, concurrent chemotherapy, side-effects of treatment, and survival data were retrieved. Follow-up status was updated in December 2018. The following procedure was followed for simulation, target volume delineation, planning and RT delivery:

\section{Simulation}

Patients were simulated on a wing board and a free breathing CECT of whole abdomen (from T6 to L5) with $100 \mathrm{ml}$ oral contrast, at $5 \mathrm{~mm}$ slice thickness (on Somatom Sensation Open CT scanner, M/s Siemens Medical System, Germany). This data was imported into the treatment planning system (Eclipse).

\section{Target volume (TV) delineation}

TV were defined according to ICRU report 62 for the two techniques.

Clinical TV (CTV) was derived by merging clinical target volume of GB bed (CTV GB) and lymph nodes (CTV LN).

CTV_GB included the hypo-density in the excised gallbladder bed (with clips if available) taking into consideration the preoperative tumour extent and $0.5 \mathrm{~cm}$ margin around it.

CTV_LN (LFRT): included abdominal LN station 5, 6,9,12 (LFRT, Figure (2) b).

CTV_LN (EFRT) (Figure 2a): included abdominal LN station 5, 6,8,9,12,13 and 16

-Attempt was made to identify and include the choledocho-jejunal anastomosis, and segment IV b of liver in the CTV, because of chances of microscopic spread of $\mathrm{GBC}$ in segment IVb of liver.

Planning target volume (PTV) was $1 \mathrm{~cm}$ expansion around CTV in all directions except craniocaudally (1.5 $\mathrm{cm}$ to account for respiratory motion) and $7 \mathrm{~mm}$ posteriorly.

Organs at risk (OAR): included liver, bilateral kidneys and spinal cord.

\section{Three- Dimensional Conformal Radiotherapy (3DCRT) planning}

PTV was treated with coplanar 3D conformal fields shaped to deliver the specified dose using a combination of 6 and 15 MV X-rays. The prescribed dose of radiotherapy to a reference point within the PTV was 50.4 Gy in 28 fractions. The treatment plan used for each patient was based on an analysis of the volumetric dose of the PTV and OAR. Normal tissue constraints were 18 Gy (median dose) for both kidneys, 30 Gy (mean liver dose) and 45 Gy (maximum spinal cord dose). Portal image of orthogonal images that localized the isocentre of planned beams was obtained on the first day of treatment only and any disparity more than the tolerance limit of $5 \mathrm{~mm}$ was corrected.

Concurrent 5-fluorouracil (cCRT: concurrent chemo radiation) was administered as a bolus of $500 \mathrm{mg}$, day 1-3 and day 29-32 (3 successive days during Week 1and 5 of RT) as tolerated. If the first cycle resulted in grade 3 diarrhoea then the dose was reduced by $10 \%$ in the next cycle. Side-effects due to concurrent chemo-radiotherapy were recorded according to CTCAE-v 3.

\section{Follow-up}

Follow-up was done by clinical examination and ultrasound whole abdomen, 3 monthly for 2 years and thereafter 6 monthly. LRR was defined as recurrence in the GB bed and regional lymph nodes. Distant metastases (DM) was defined as recurrence in distant organs. Any evidence of LRR or DM was confirmed by CECT abdomen and fine needle aspiration cytology. Overall survival (OS) and disease-free survival (DFS) were estimated using Kaplan-Meier method using SPSS v 15.

\section{Results}

The demographic characteristics of patients $(n=60)$ were: median age 53 years (range 27-77 yrs.), 18 males (30\%) and 42 females (70\%), stage II: 5 (11.1\%), 
Table 1. Patient Characteristics

\begin{tabular}{|c|c|c|}
\hline \multirow[t]{2}{*}{ Clinical and treatment characteristics } & Localised field (LFRT) & Extended field (EFRT) \\
\hline & $\mathrm{N}=30, \%$ & $\mathrm{~N}=30, \%$ \\
\hline Age in median (IQR) & $52(42-57)$ & $54(41-70)$ \\
\hline Sex (female) & $27: 73$ & $34: 66$ \\
\hline \multicolumn{3}{|l|}{ Surgical technique $(\%)$} \\
\hline Extended cholecystectomy & $20(66.7)$ & $30(100)$ \\
\hline Cholecystectomy with hepatectomy & $7(23.3)$ & - \\
\hline Cholecystectomy with Hepato-pancreaticoduodenectomy & $3(10)$ & - \\
\hline \multicolumn{3}{|l|}{$\mathrm{T}$ status $(\%)$} \\
\hline 1 & $1(3.3)$ & - \\
\hline 2 & $16(53.3)$ & $12(40)$ \\
\hline 3 & $13(43.3)$ & $16(53.3)$ \\
\hline 4 & - & $2(6.7)$ \\
\hline \multicolumn{3}{|l|}{$\mathrm{N}$ status $(\%)$} \\
\hline 0 & $8(26.7)$ & $10(33.3)$ \\
\hline 1 & $17(56.7)$ & $8(26.7)$ \\
\hline 2 & $5(16.7)$ & $12(40)$ \\
\hline \multicolumn{3}{|l|}{ Stage group (\%) } \\
\hline 1 & $1(3.3)$ & - \\
\hline 2 & $3(10)$ & $2(6.7)$ \\
\hline 3 & $26(86.7)$ & $20(66.7)$ \\
\hline 4 & - & $8(26.7)$ \\
\hline \multicolumn{3}{|l|}{ Grade $(\%)$} \\
\hline WD & $22(73.3)$ & $18(60)$ \\
\hline $\mathrm{PD}$ & $8(27.3)$ & $12(40)$ \\
\hline \multicolumn{3}{|l|}{ Resection status (\%) } \\
\hline R0 & $13(43.3)$ & $16(53.3)$ \\
\hline $\mathrm{R} 1$ & $17(56.7)$ & $14(46.7)$ \\
\hline LVI (\%) & $5(16.7)$ & 0 \\
\hline PNI (\%) & $4(13.3)$ & 0 \\
\hline
\end{tabular}

IIIa: 11 (24.4\%), IIIb: 26 (57.8\%), stage IV: 3(6.6\%) (Table 1). Forty-eight patients $(80 \%)$ underwent extended cholecystectomy (EC), nine (15\%) underwent EC with right hepatectomy and lymphadenectomy and three (5\%) underwent EC with pancreato-duodenectomy. 30 patients were treated with LFRT (2007-2010) and 30 with EFRT (2011-2014). The demographic features and tumour and treatment related factors were well balanced in both the arms. The median radiotherapy dose was $50.4 \mathrm{~Gy}$ (range 45Gy - 50.4 Gy). 2 patients could not tolerate concurrent 5-FU, hence the second cycle of 5-FU was not given subsequently. Those who were elderly (age $>$ 70 years were not offered concurrent chemotherapy, $n=3$ ).
$10 \%$ and $15 \%$ patients experienced grade 2 emesis and dyspepsia in LFRT and EFRT respectively. Though grade 3 acute toxicity was not seen during treatment, 2 patients died, one due to subacute intestinal obstruction 6 months following treatment due to local recurrence and in second the cause of death could not be ascertained. No grade 3 late morbidity was reported till the time of last follow-up.

The median follow up for all patients was 44 months (IQR 36 - 120 months) and 68 months for those alive (36-120 months). Ten percent patients $(6 / 60)$ were lost to follow-up. There was significant difference in median overall and disease free survival time between the two groups. The 5 year overall survival (OS) rates was $80 \%$

Table 2. Local Recurrence and Survival in LFRT and EFRT

\begin{tabular}{lccc}
\hline Outcomes & Localised field $(\mathrm{n}=30)$ & Extended field $(\mathrm{n}=30)$ & $\mathrm{p}$-value \\
\hline \#Overall survival (in months) & $34(4-64)$ & $\mathrm{NR}$ & 0.03 \\
\#Disease free survival (in months) & $25(11-50)$ & $\mathrm{NR}$ & 0.03 \\
Local recurrence & $12(40 \%)$ & $4(13.3 \%)$ & 0.18 \\
Distant metastases & $8(26.7 \%)$ & $0(0 \%)$ & 0.01 \\
\hline
\end{tabular}

\#Data presented in median (IQR), compared by Log rank test 


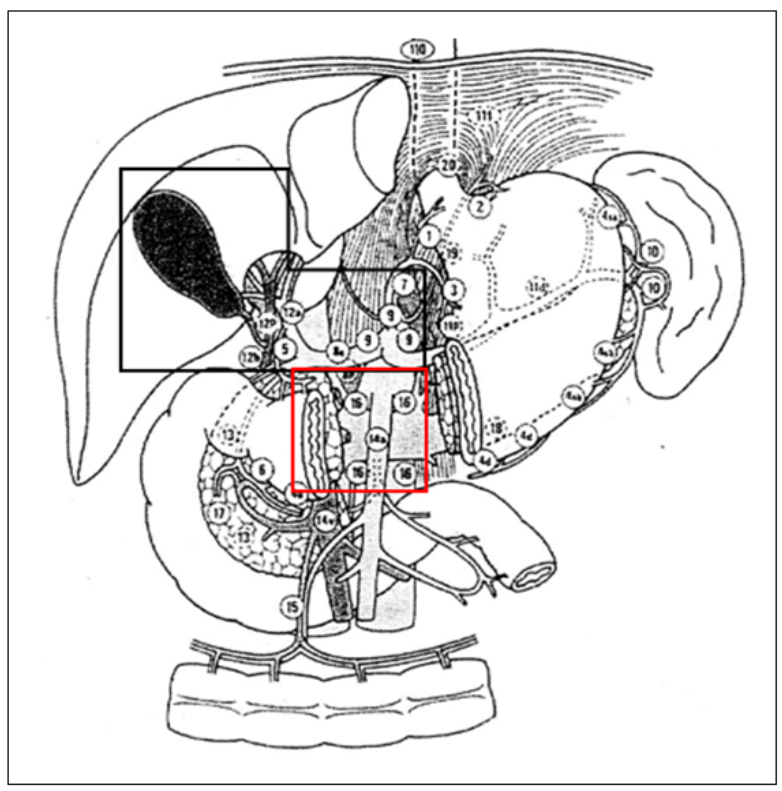

Figure 1. LFRT (black box): Includes Postoperative GB bed, Peri-portal, Common Hepatic Artery and Coeliac lymph-nodes, EFRT (black + red box): Postoperative GB bed, Peri-portal, Common Hepatic Artery, Coeliac, retro-pancreatic Nodes, Anterior and Posterior Pancreatico-duodenal Nodes, and Para-aortic Lymph-nodes.

and $42 \%$ for EFRT and LFRT respectively ( $p=0.01$, Table 2 , Figure 3 (a)). The 5 year disease free survival (DFS) was $80 \%$ and $40 \%$ for EFRT and LFRT respectively $(p=0.01$, Figure $3(b))$. This benefit was seen irrespective of resection and nodal status. Overall $37.5 \%(16 / 60)$ patients developed recurrence ( $13.3 \%$ vs $40 \%$ in EFRT and LFRT). While $80 \%$ of patients with LRR in LFRT had R1 and node positive disease, both patients with LRR in EFRT had R1 disease and one was lost to follow-up. The incidence of distant metastases (DM) was $26.6 \%$ in LFRT and $0 \%$ in EFRT.

\section{Discussion}

Our results demonstrate that irradiation of para-aortic lymph nodes improves LRR, DFS and OS irrespective of the resection and nodal status. Though patient numbers are small, the improvement in survival rates are optimistic and suggests inclusion of para-aortic node in all patients of $\mathrm{GBC}$ after RS. This also reiterates the fact that careful and consistent CTV delineation resulted in decrease of LRR rates from $40 \%$ in LFRT to $13 \%$ in EFRT. Our results also suggest that better LRR results in decrease in DM rates. The GBC audit of National Cancer Database of 4775 patients showed only early survival benefit (3 year OS of $39.9 \%$ ) in patients who received CTRT as compared to those who did not ( 3 year OS of $6.8 \%$ ). The authors contested that the poor outcome was due to the inclusion of a mixed bag of patients (in-adequate surgery [simple/ partial cholecystectomy: $26.4 \%$ ] and adequate surgery [total cholecystectomy: $27.1 \%$ ]) and that the benefit of adjuvant therapy should be assessed only in patients who have undergone RS [3]. Our data shows that with EFRT the 5 year OS rose to $80 \%$ and this benefit is sustainable. In a multi-institutional audit of adjuvant CTRT (EFRT) in GBC patients (in those with node positive, high grade and LVI), the 5 year OS was $44.9 \%$ [4]. Based on the absence of late morbidity in liver, kidney and small bowel, irradiation of para-aortic nodes seems to be safe.

Our study emphasizes that standardisation of CTV delineation improves local control. Poor LRR with LFRT was due to non-inclusion of superior mesenteric nodes, para-aortic nodes, due to lack of availability of guidelines at that time. With availability of RTOG guidelines for postoperative pancreatic cancers, we adopted similar LN-CTV delineation volumes in GBC. We propose to draw two clinical target volumes, the GB bed (CTV_GB) and the lymphatic CTV (CTV_LN) and then merge both the volumes to form the CTV. Based on the fact that GBC spreads by direct infiltration to adjacent liver segments IV and V (34-83\%), the cystic duct, hepatico - choledochal duct (6-57\%) and the peritoneum of the hepatoduodenal ligament, these structures should be included along with clips at the liver resection margin (if available) to form the CTV_GB [12]. The role of surgical clips in aiding CTV delineation for postoperative GBC is not available in literature, but it is intuitive that it will improve the accuracy of CTV delineation as seen in other tumors. The number, site and type of clips to be inserted (to differentiate from haemostatic clips) needs to be standardised. The preoperative CT scans should be fused with the simulation scans, but it is not routinely advocated because the postoperative anatomy varies considerably from preoperative anatomy, the preoperative imaging is not obtained on a flat table or with appropriate patient positioning, the imaging sets may have been obtained in different phases of respiration, and there may be significant change in weight and body habitus after RS. Since no histopathological studies of microscopic spread are available in the literature for GBC, an arbitrary 0.5 $\mathrm{cm}$ margin was given around the above target volume to form CTV_GB.

To delineate the CTV_LN, familiarity with lymphatic dissemination is essential. The lymphatic spread in GBC cancers follows an intrahepatic and an extrahepatic path. The intrahepatic spread occurs along lymph vessels in adjacent liver segments IV and V towards the lymphatic tissue in liver hilum and the hepatoduodenal ligament lymphatics. The extrahepatic lymphatic spread follows the sub-serosal lymphatic tissue of the gallbladder and the cystic duct to the hepatoduodenal and pancreatoduodenal lymphatics (lymph-node stations 8, 9 and 12). The hepatoduodenal lymphatic network drains to the superior, anterior and posterior pancreato-duodenal lymph nodes, aorto - caval nodes near the left renal vein, superior mesenteric artery, nodes along the hepatic artery and coeliac trunk (lymph-node stations 5,6,13 and 16) [13]. Hence all the above LN stations were included and merged with CTV_GB bed to form the CTV in EFRT. The altas for CTV definition for locally advanced GBC published this year also suggests similar target volumes [14].

APTV margin of $1 \mathrm{~cm}$ (except cranio-caudal margin of 


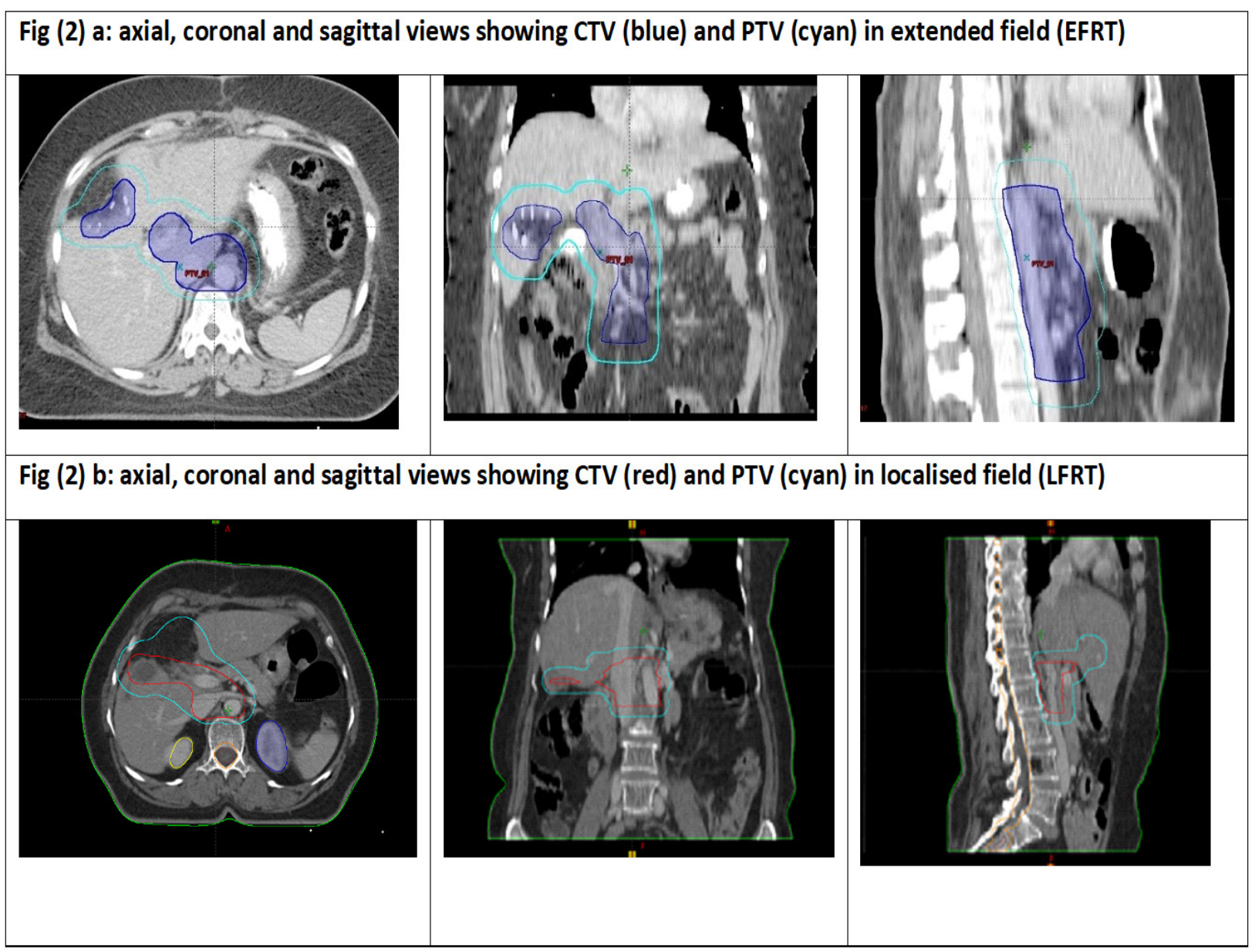

Figure (2). a, Clinical Target Volume (CTV) and Planning Target Volume (PTV) in Extended Field RT (EFRT) in Axial, Sagittal and Coronal View; b, Clinical target volume (CTV) and planning target volume (PTV) in localised field RT (LFRT) in axial, sagittal and coronal view.



Figure 3. a, Overall Survival with LFRT and EFRT; b, Disease Free Survival with LFRT and EFRT

$1.5 \mathrm{~cm}$ for respiratory motion and $7 \mathrm{~mm}$ posteriorly) was given to account for setup errors based on our departmental data on chest tumours positioned on a wing board [15]. Due to lack of facility of 4D-CT, individual respiratory motion could not be accounted for though data from 4D CT in liver stereotactic treatment does reveal that compared to conventional PTV, the use of 4D CT-based
PTVs with individual margins significantly reduces normal tissues being unnecessarily irradiated and also contributes to reducing the risk of missing targets for tumours with large motion [16-17].

Our findings validates the suggestions of the systematic pathologic analysis for inclusion of abdominal LN station 5,6,8,9,12,13,16 for TV delineation in GBC, 
as it has shown better outcome [10]. In the absence of any randomised study comparing LFRT and EFRT, we suggest that EFRT can be practiced to achieve low LRR and DM rates. Since resectable GBC are only $10 \%$ of the GBC patients presenting in clinic, conducting a randomised study to compare both the techniques is difficult because of likelihood of low accrual.

The limitations of our study is the small sample size which can be explained by the rarity of early stage GBC presentation. The sample size explains the demographic and clinical differences between the two groups. Propensity score analysis to account for these differences was not undertaken due to the small sample size. Though literature suggests the lymph-node stations to be irradiated for biliary cancers, outcomes with RT based on localised and extended field RT is not available in the literature in this disease with high mortality.

In conclusions, based on our findings, we conclude that EFRT reduces LRR and improves survival in patients with absence of lympho-vascular space invasion (LVI) and peri-neural invasion (PNI). This observation is hypothesis generating. A prospective randomized trial with EFRT is desirable to validate our observations.

\section{Acknowledgments}

Not applicable.

\section{Authors' contributions:}

All authors read and approved the final manuscript

\section{Funding}

Non-funded

\section{Availability of data and materials}

Datasets used and/or analyzed in the current study are available from the corresponding author on reasonable request.

\section{Ethics approval and consent to participate}

Institutional Ethics Committee

\section{Consent for publication}

Not applicable.

\section{Competing interests}

The authors declare that they have no competing interests.

\section{References}

1. Randi G, Franceschi S, La Vecchia C. Gallbladder cancer worldwide: Geographical distribution and risk factors. International Journal of Cancer. 200604 01;118(7):15911602. https://doi.org/10.1002/ijc.21683

2. Jarnagin WR, Ruo L, Little SA, Klimstra D, D’Angelica M, DeMatteo RP, Wagman R, Blumgart LH, Fong Y. Patterns of initial disease recurrence after resection of gallbladder carcinoma and hilar cholangiocarcinoma. Cancer. 2003 Oct 03;98(8):1689-1700. https://doi.org/10.1002/cncr.11699

3. Mantripragada KC, Hamid F, Shafqat H, Olszewski AJ.
Adjuvant Therapy for Resected Gallbladder Cancer: Analysis of the National Cancer Data Base. Journal of the National Cancer Institute. 2016 Oct 05;109(2):djw202. https://doi.org/10.1093/jnci/djw202

4. Kim Y, Amini N, Wilson A, Margonis GA, Ethun CG, Poultsides G, Tran T, Idrees K, Isom CA, Fields RC, Krasnick B, Weber SM, Salem A, Martin RCG, Scoggins C, Shen P, Mogal HD, Schmidt C, Beal E, Hatzaras I, Shenoy R, Cardona K, Maithel SK, Pawlik TM. Impact of Chemotherapy and External-Beam Radiation Therapy on Outcomes among Patients with Resected Gallbladder Cancer: A Multi-institutional Analysis. Annals of Surgical Oncology. 201605 11;23(9):2998-3008. https://doi. org/10.1245/s10434-016-5262-8

5. Agrawal S, Gupta PK, Rastogi N, Lawrence A, Kumari N, Das KJM, Saxena R. Outcomes of Adjuvant Chemoradiation and Predictors of Survival After Extended Cholecystectomy in Gall Bladder Carcinoma: a Single Institution Experience from an Endemic Region. Journal of Gastrointestinal Cancer. 2014 Dec 16;46(1):48-53. https://doi.org/10.1007/s12029014-9676-x

6. Abrams RA, Winter KA, Regine WF, Safran H, Hoffman JP, Lustig R, Konski AA, Benson AB, Macdonald JS, Rich TA, Willett CG. Failure to Adhere to Protocol Specified Radiation Therapy Guidelines Was Associated With Decreased Survival in RTOG 9704-A Phase III Trial of Adjuvant Chemotherapy and Chemoradiotherapy for Patients With Resected Adenocarcinoma of the Pancreas. International Journal of Radiation Oncology*Biology*Physics. 2012 02;82(2):809816. https://doi.org/10.1016/j.ijrobp.2010.11.039

7. Gold DG, Miller RC, Haddock MG, Gunderson LL, Quevedo F, Donohue JH, Bhatia S, Nagorney DM. Adjuvant Therapy for Gallbladder Carcinoma: The Mayo Clinic Experience. International Journal of Radiation Oncology*Biology*Physics. 2009 09;75(1):150-155. https:// doi.org/10.1016/j.ijrobp.2008.10.052

8. Yamaguchi K, Chijiwa K, Shimizi S. Anatomical limit of extended cholecystectomy for gallbladder carcinoma involving the neck of the gallbladder. Int Surg. 1998;83:21-3.

9. Todoraki T, Kawamoto T, Otsuka M, Koike N, Yoshida S, Takada Y. Benefits of combining radiotherapy with aggressive resection for stage IV gallbladder Cancer. Hepatogastroenterology. 1999;46:1585-91.

10. González ME, Giannini OH, González P, Saldaña B. Adjuvant radio-chemotherapy after extended or simple cholecystectomy in gallbladder cancer. Clinical and Translational Oncology. 2011 07;13(7):480-484. https://doi. org/10.1007/s12094-011-0685-y

11. Clinical Target Volume in Biliary Carcinoma: A Systematic Review of Pathological Studies. Anticancer Research. 201703 17;37(3):955-962. https://doi.org/10.21873/ anticanres.11404

12. Goodman KA, Regine WF, Dawson LA, Ben-Josef E, Haustermans K, Bosch WR, Turian J, Abrams RA. Radiation Therapy Oncology Group Consensus Panel Guidelines for the Delineation of the Clinical Target Volume in the Postoperative Treatment of Pancreatic Head Cancer. International Journal of Radiation Oncology*Biology*Physics. 2012 07;83(3):901908. https://doi.org/10.1016/j.ijrobp.2012.01.022

13. Fahim RB, McDonald JR, Richards JC, Ferris DO. Carcinoma of the Gallbladder. Annals of Surgery. 1962 07;156(1):114124. https://doi.org/10.1097/00000658-196207000-00021

14. Bisello S, Renzulli M, Buwenge M, Calculli L, Sallustio G, Macchia G, Deodato F, Mattiucci G, Cammelli S, Arcelli A, Giaccherini L, Cellini F, Brandi G, Guerri S, Cilla S, Golfieri R, Fuccio L, Morganti A, Guido A. An atlas for clinical target 
volume definition, including elective nodal irradiation in definitive radiotherapy of biliary cancer. Oncology Letters. 2018 Nov 28;. https://doi.org/10.3892/ol.2018.9774

15. Raza W, Agarwal S, Maria Das KJ, Senthil Kumar SK, Lal P. Comparison of set-up errors by breast size on wing board by portal imaging. Reports of Practical Oncology \& Radiotherapy. 2016 09;21(5):447-452. https://doi. org/10.1016/j.rpor.2016.04.001

16. Gabryś D, Kulik R, Trela K, Ślosarek K. Dosimetric comparison of liver tumour radiotherapy in all respiratory phases and in one phase using 4DCT. Radiotherapy and Oncology. 2011 09;100(3):360-364. https://doi. org/10.1016/j.radonc.2011.09.006

17. Li F, Li J, Xing J, Zhang Y, Fan T, Xu M, Shang D, Liu T, Song J. Analysis of the advantage of individual PTVs defined on axial 3D CT and 4D CT images for liver cancer. Journal of Applied Clinical Medical Physics. 2012 Nov;13(6):62-71. https://doi.org/10.1120/jacmp.v13i6.4017

\section{(c) (1) (9)}

This work is licensed under a Creative Commons AttributionNon Commercial 4.0 International License. 